\title{
Solar Wind Control of the Size and Shape of the Magnetosphere
}

\author{
D. H. FAIRFIELD \\ Laboratory for Extraterrestrial Physics, Nasa Goddard Space Flight Center, Greenbelt, MD 20771, U.S.A.
}

(Received August 18, 1990; Revised January 29, 1991)

\begin{abstract}
The position of the earth's magnetopause is controlled by the kinetic pressure of the solar wind and further influenced by the north-south component of the interplanetary magnetic field. On average the distance to the subsolar magnetopause is $\sim 10 R_{\mathrm{E}}$, but this distance varies with the solar wind pressure during the 11 year sunspot cycle. Maximum solar wind pressure occurs during the declining phase of the cycle and compresses the average subsolar magnetopause inside $10 R_{E}$ while minimum pressure occurs during solar maximum, allowing expansion of the magnetopause outward toward $11 \mathrm{R}_{\mathrm{E}}$. The northsouth component of the IMF moves the subsolar magnetopause inward or outward approximately .25 $R_{E}$ for each $1 \mathrm{nT}$ unit of negative or positive field strength, with the magnetotail expanding or contracting to contain the flux transported into the tail. Other observations suggest that the distant tail (1) may be flattened into an elliptical cross section with major axis aligned with the $Y Z$ component of the IMF and (2) is probably twisted by the torque exerted by interplanetary field lines connected to the tail lobes. Recent evidence suggests that reorientations of the upstream foreshock may produce density enhancements of a few minutes duration that impinge on the magnetopause and compress the magnetosphere.
\end{abstract}

\section{Introduction}

Diversion of the solar wind around the earth and the confinement of Earth's magnetic field to the region known as the magnetosphere is a fundamental problem of solar terrestrial physics. Early studies of the position and shape of the bounding surface of the magnetosphere, the magnetopause, have been summarized by FAIRFIEI.D (1971) who consolidated 474 crossing of the boundary obtained from 6 different spacecraft during the interval 1963-1968. This data set was subsequently extended by HoLzER and SLAVIN (1978), FORMISANO et al. (1979), and most recently by SIBECK et al. (1991). This latest study increases the number of collected crossings to 1820 and is particularly effective in illustrating the important ways the solar wind controls the magnetosphere. Since the magnetopause has now been studied over nearly three solar cycles, it is an appropriate time to review our understanding of how and why the magnetopause position varies.

\section{Solar Wind Pressure Control of the Magnetopause}

Figure 1 (SIBECK et al., 1991) illustrates the observed magnetopause positions in a coordinate system where $X$ points toward the sun and $R=\left(Y^{2}+Z^{2}\right)^{1 / 2}$. The aberration due to the earth's motion about the sun was removed from the individual measurements and symmetry about the earth-sun line was the invoked. Much of the scatter in the 


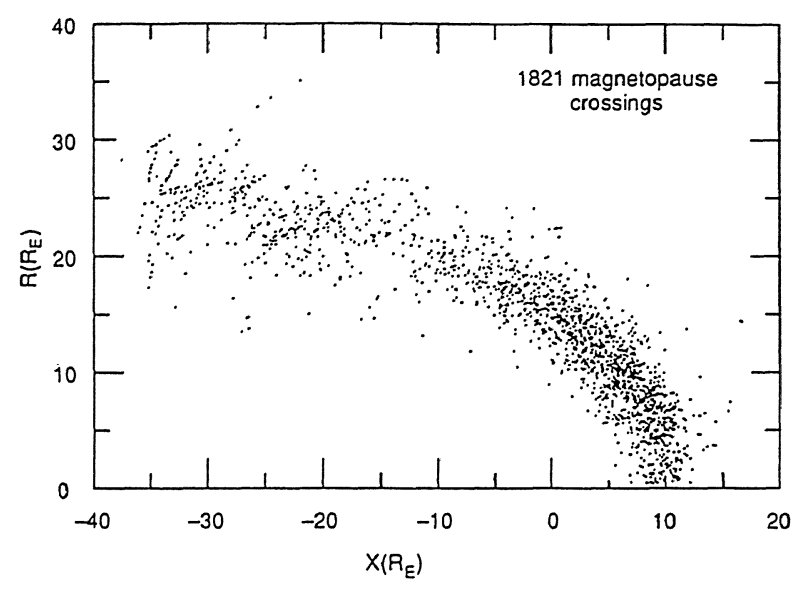

Fig. 1. The observed magnetopause crossing locations detected by many spacecraft throughout the space age. The $X$ axis is the earth-sun line and $R$ is the distance from the $X$ axis.

magnetopause locations in Fig. 1 is presumably due to day-to-day changes in the pressure of the solar wind. To investigate this point the authors assumed the standard model where solar wind pressure is balanced by the internal field

$$
k n M V^{2} \cos ^{2} \theta=(2 f B)^{2} / 8 \pi
$$

where $n$ is the solar wind density, $M$ is the proton mass, $V$ is the solar wind velocity, $\theta$ is the angle the solar wind makes with the normal to the magnetopause, $B$ is the field strength just inside the magnetopause, and $f^{2} k=1.69$ as determined by HoLzER and SLAVIN (1978) (see MACEK (1988) for a full review of this theoretical approach). Note that although the magnetosheath pressure is directly involved in the pressure balance at the magnetopause, solar wind pressure is normally used in place of the less-easilymonitored magnetosheath pressure; apparently momentum flux is conserved as the directed solar wind kinetic energy is converted into thermal and field energy at the bow shock so that solar wind pressure is an adequate proxy for solar wind pressure. Standard theory then assumes that the magnetosphere field at the subsolar point is given by the dipole field $B=H_{0} / R^{3}$ where $H_{0}$ is the equatorial field strength at the earth's surface. Inserting $B$ in Eq. (1) yields the well known inverse sixth power relation between pressure and $R$ at the subsolar magnetopause point $(\cos \theta=1)$

$$
R=\left[\left(f^{2} / k\right)\left(H_{0}^{2} / 2 \pi n M V^{2}\right)\right]^{1 / 6} .
$$

In their analysis Sibeck et al. first associated the measured magnetopause positions with simultaneous hourly average measurements of the solar wind. Next they separated the data points into five groups according to solar wind pressure $(0.54-0.87,0.87-1.47$, $1.47-2.6,2.6-4.9,4.9-9.9 \mathrm{nPa}$ ), with the pressure bins chosen to give average magnetopause positions separated by $1 \mathrm{R}_{\mathrm{E}}$ if Eq. (2) is correct. Then they independently fit the data in the five groups to the equation $R^{2}=a X^{2}+b X+c$ producing the 5 curves shown in Fig. 2 (SIBECK et al., 1991). The predicted $1 \mathrm{R}_{\mathrm{E}}$ separations of the curves are indeed 


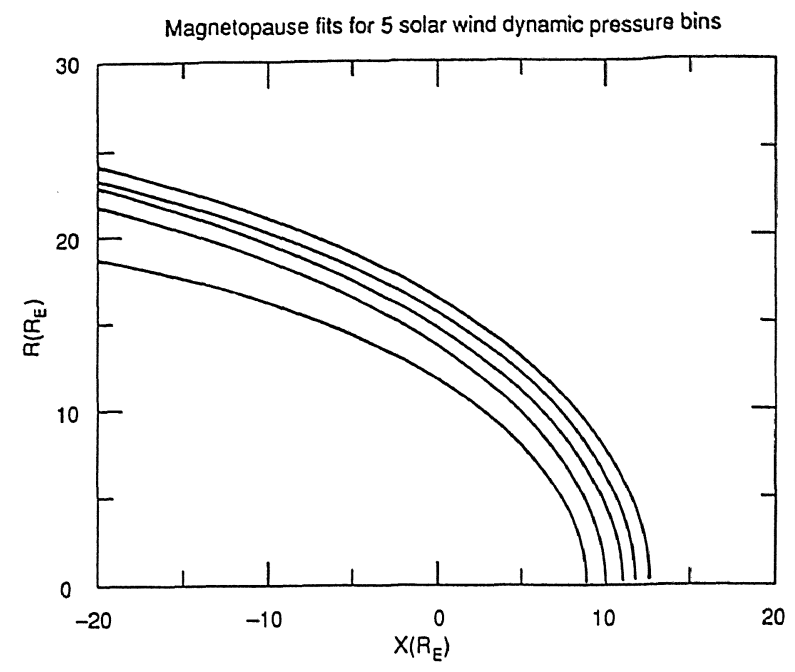

Fig. 2. Average magnetopause curves determined from least-squares fitting five separate data sets corresponding to different bins of solar wind pressure (SiBECK et al., 1991).

obtained, thus supporting the validity of Eq. (2) and justifying the choice of the constant $f^{2} / k=1.69$. Furthermore it is noted that the shapes of the curves are self-similar, indicating that the magnetopause shape (i.e. the ratio of the distances from the earth to the magnetopause in the noon and dawn-dusk meridians) does not change as function of solar wind pressure.

If indeed the shape and position of the magnetopause is controlled by solar wind pressure, it is of interest to inquire into the variability of this pressure, both on a day-today basis and over the solar cycle. Figure 3 presents annual averages of relevant solar wind quantities measured by spacecraft throughout the space age and supplied by J. W. King. Quantities shown are the June values of the 12 month running averages of the sunspot number $R$ (NOAA Solar Geophysical Data Prompt Reports), the annual averages of hourly values of solar wind kinetic pressure $n M v^{2}$, solar wind density $n$, solar wind velocity $v$, and the percent solar wind coverage for each year. The figure extends an earlier figure (FAIRFIELD, 1979) and supports the earlier suggestion that there is a solar cycle dependence in the solar wind pressure exerted on the magnetopause. A scale to the right of the pressure trace indicates the expected magnetopause position as calculated by Eq. (2). The data are in agreement with the average subsolar magnetopause position of 10.8 determined in Fairfield's 1971 study, but they also show that during the subsequent 20 years an average value near 10.0 is more appropriate. It is interesting to note that minimum average solar wind pressures occur near solar maximum and maximum pressures occur during the declining phase of the solar cycle when high speed streams are most prevalent (e.g. PIzzo, 1986). Figure 3 shows that the excursion of annual average subsolar magnetopause position over the solar cycle is at least $1 \mathrm{R}_{\mathrm{E}}$ in magnitude. The above discussion should be qualified, however, by citing a discussion of KING (1991) who intercalibrated various solar wind data sets. He notes that there was only minimal overlap between spacecraft making measurements before and after 1971 and hence densities could be too low or high before or after this date. King further cites a private 


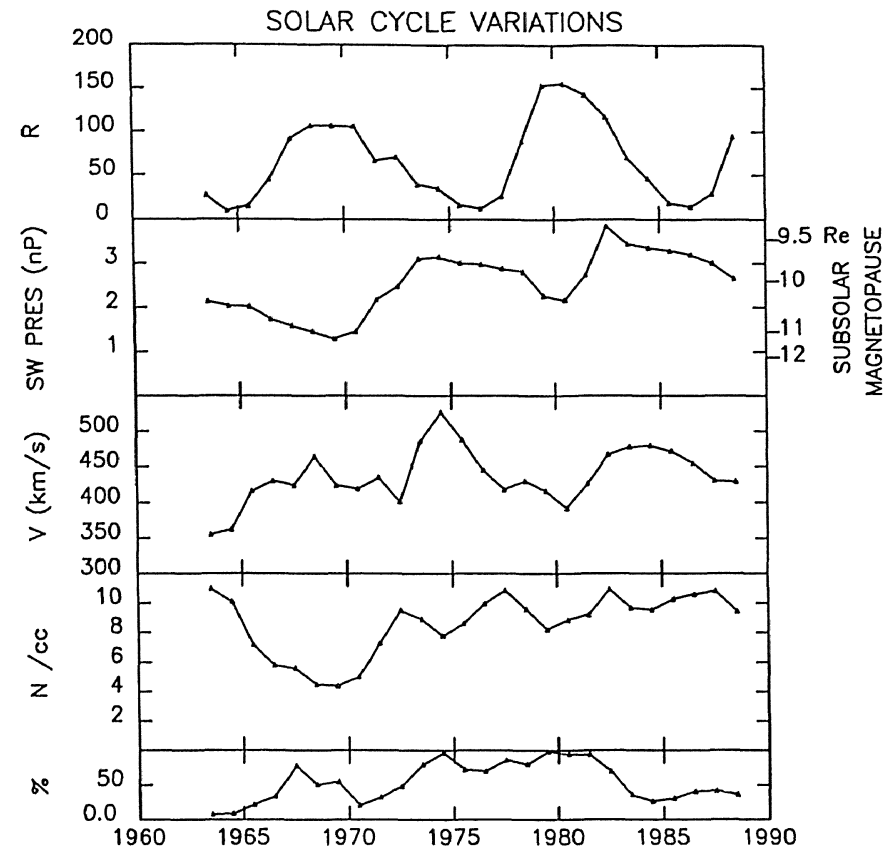

Fig. 3. Annual averages of solar wind quantities throughout the satellite era. Solar wind kinetic pressure exhibits a solar cycle variation with minimum pressure at solar maximum (maximum sunspot number $R$ ) and maximum during the declining phase of the solar cycle. Predicted subsolar magnetopause distances for various solar wind pressures are shown by the scale on the right.

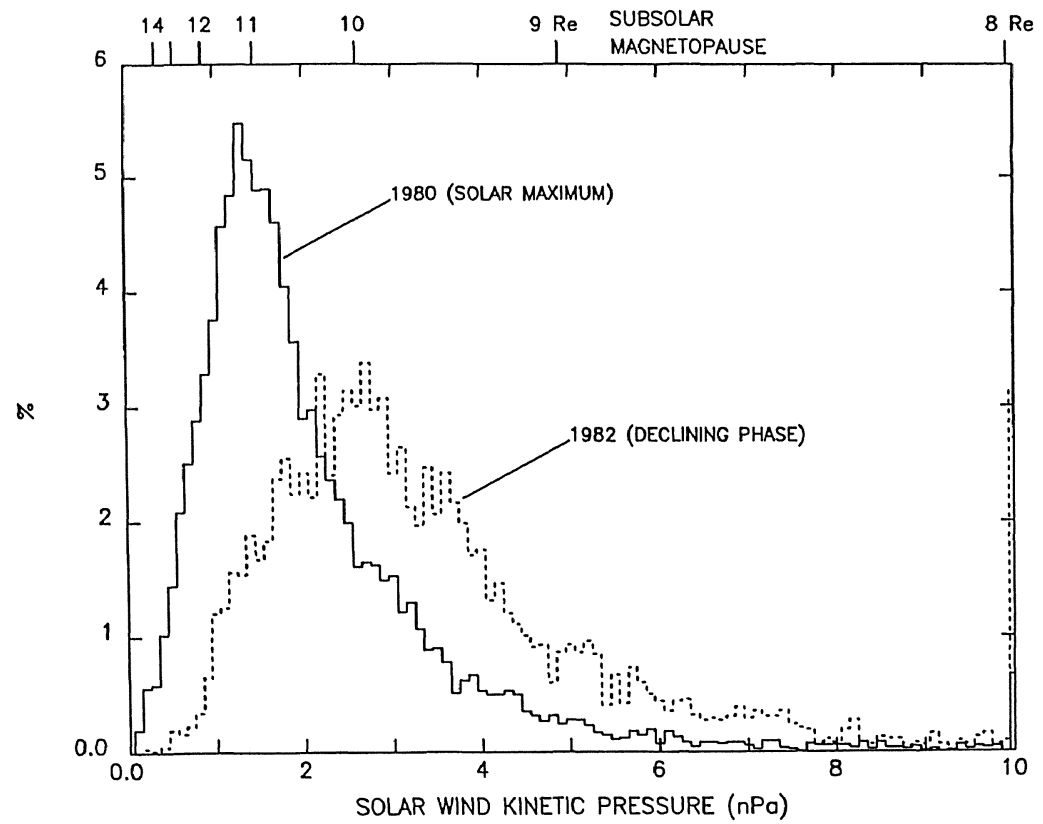

Fig. 4. Distributions of solar wind pressure during years of minimum pressure (1980) and maximum pressure (1982). Corresponding subsolar magnetopause positions are indicated by the scale at the top. 
communication from Sibeck who after analyzing magnetopause positions before and after 1971 , found that pressures prior to this year might be low by perhaps $60 \%$. If indeed pressures should be increased prior to 1971, this would decrease the amplitude of the solar cycle variation, but not eliminate its presence.

To further investigate the hour-to-hour variations in pressure during individual years, the low pressure, solar maximum year of 1980 was selected to contrast with the high pressure year of 1982. Distributions of solar wind pressure for these years are shown in Fig. 4 with the subsolar magnetopause scale included at the top. As expected, there is considerable scatter about the annual averages of 2.2 and 3.8 for 1980 and 1982 respectively. It is interesting the 1982 not only has higher pressures due to more frequent high speed streams, but it also has substantially fewer hours with pressure below $1 \mathrm{nPa}$. Note that Eq. (2) predicts that a pressure of $31 \mathrm{nPa}$ is needed to compress the magnetopause to geosynchronous orbit, a value that occurs very infrequently according to Fig. 4. A southward IMF $B_{z}$ is needed to move the magnetopause very close to the earth and this is the second major factor that controls the magnetopause position.

\section{Interplanetary Magnetic Field $B_{z}$ Control of the Magnetopause Position}

For many years it has been known that the north-south component of the interplanetary magnetic field also controls the position of the magnetopause (AUBRY et al., 1970; FAIRFIELD, 1971; HOLZER and SLAVIN, 1978). Again the recent work of Sibeck et al. very clearly demonstrates this effect. When these authors fit their positional data after binning it into six, $2 \mathrm{nT}$ groupings according to the hourly IMF $B_{z}$ values ranging from +6 to $-6 \mathrm{nT}$, they obtained the curves shown in Fig. 5. The subsolar point

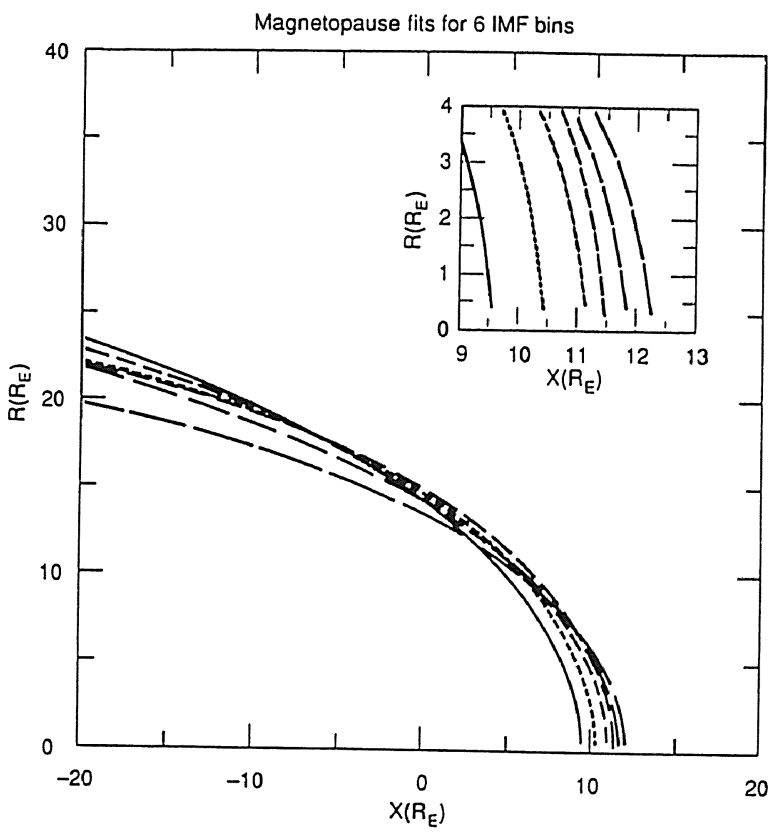

Fig. 5. Average magnetopause curves determined from least-squares fitting five separate data sets corresponding to different bins of interplanetary magnetic field $B_{z}$ (SIBECK et al., 1991). 
determined by fitting the data moves inward from $12.2 \mathrm{R}_{\mathrm{E}}$ for the largest positive $B_{z}$ to 9.6 $\mathrm{R}_{\mathrm{E}}$ for the most negative $B_{z}$. Now the curves are no longer self similar with the nightside magnetopause distance generally increasing as the subsolar point decreases, thus indicating that the flux removed from the dayside is transported to the magnetotail.

This concept of transferring flux from the dayside to the nightside is very old and has been discussed previously by many authors (e.g. HOLZER and SLAVIN, 1978 and references therein). Experimental verification in addition to the dayside magnetopause position studies mentioned above include BURCH (1972), who observed southward motion of the polar cusp in the preseuce of a southward $B_{z}$, MAEZAWA (1975) who detected outward motion of the tail magnetopause before the expansive phase onset of substorms and inward motion after onset, and FAIRFIELD (1985) who determined the time-varying flaring angle of the tail by monitoring the solar wind pressure and the field strength in the magnetotail. As flux is added to the tail, the tail radius increases, thus increasing the flaring angle of the tail and the component of pressure incident on the boundary (see Eq. (1)). This process increases the tail lobe field strength and stores energy in the tail. FAIRFIELD et al. (1989) recently used multiple spacecraft to study an event that demonstrated these various effects and in addition detected increases and decreases in the area of the polar cap in concert with tail lobe field strength changes (see also FRANK and CRAVEN, 1989).

This IMF control of the magnetopause also explains the occurrence of the magnetopause inside of geosynchronous orbit. Two separate studies indicate that such events occur $0.3 \%$ of the time (RUSSELL, 1976) or $0.1 \%$ of the time (RUFENACH et al., 1989 ) with the different percentages apparently being due to various assumptions made about where the magnetopause could be observed. Russell found ATS 1 in the magnetosheath for a total of about 5 hours between December 1966 and December 1968 $(\sim 2.5$ hours/year) while Rufenach et al. found GOES spacecraft in the magnetosheath for 32 hours from 1978-1986 (4 hours/year). Russell's 0.3\% estimate applies to the noon sector whereas Rufenach et al.'s $0.1 \%$ determination apparently assumed equal likelihood of being in the magnetosheath throughout the entire sunward hemisphere. The fact that Rufenach et al. find their close-in crossings generally associated with southward field conditions as well as following storm sudden commencements leads to their conclusion that both pressure and $B_{z}$ effects are important in bringing the magnetopause inside of the geosynchronous location.

SIBECK et al. (1991) represent the dependence of the magnetopause subsolar location on the IMF $B_{z}$ by the equation

$$
R\left(\mathrm{R}_{\mathrm{E}}\right)=11.3+.25 \mathrm{IMF} B_{z}(\mathrm{nT})
$$

Rufenach et al., in their study of geosynchronous magnetopause crossings also obtained a constant of proportionality but with a value only about one third as large at that of SIBECK et al. (1991).

If the magnetospheric magnetic field pressure indeed balances the solar wind kinetic pressure and at the same time $B_{z}$ causes movement of the magnetopause, the magnetospheric field strength must adjust itself in response to the IMF. This idea is illustrated by Fig. 6. Here a schematic magnetospheric field equal to twice the dipole field strength is plotted as a function of radius out to a magnetopause at $10 \mathrm{R}_{\mathrm{E}}$. Now suppose that $B_{z}$ becomes negative, thus moving the magnetopause inward to $9 R_{E}$ but with no change in 


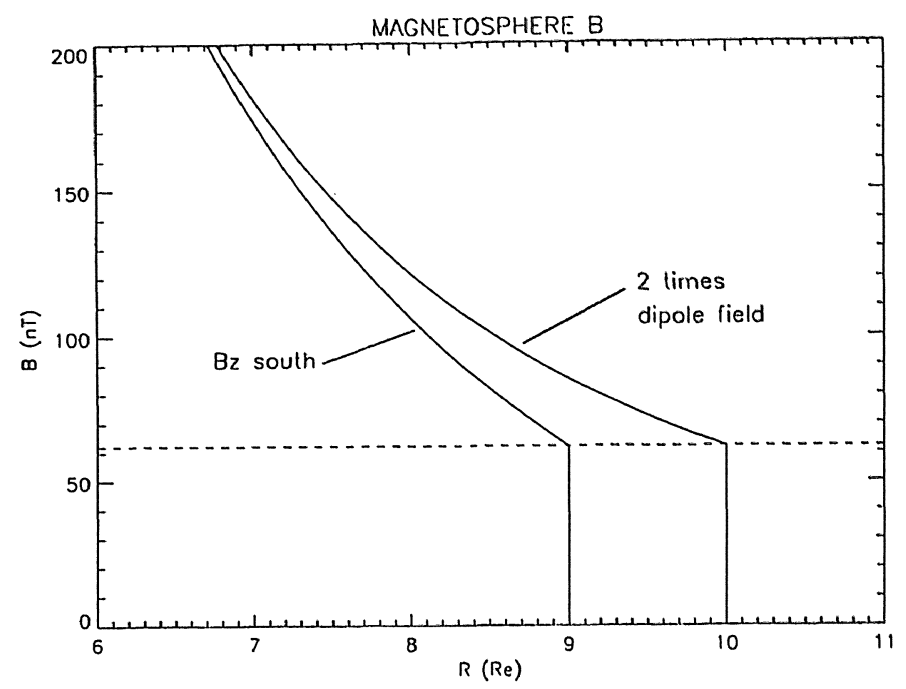

Fig. 6. Schematic representation of the earth's equatorial field strength as a function of radial distance. The variation with distance must be different if the magnetopause is at 9 or $10 R_{\mathrm{E}}$ but the solar wind pressure is the same.

solar wind pressure. The magnetosphere field at $9 \mathrm{R}_{\mathrm{E}}$ must remain at the same value it had at $10 R_{E}$ to balance the solar wind, thus necessitating a readjustment in the internal field strength such as is shown by the curve marked $B_{z}$ south. Although an increased cross-tail current could decrease this dayside field strength, MALTSEV and LYATSKY (1975) suggest that Region 1 field aligned currents in the cusp region are responsible for the decrease. This $B_{z}$ control indicates that the magnetopause position is more closely related to magnetosphere dynamics than would have been suspected for the simple self-similar compression by enhanced pressure.

\section{IMF Control of the Shape of the Magnetotail Cross Section}

For many years measurements in the cis-lunar magnetotail have been unable to clearly establish whether the tail cross section is elliptical and, if so, what is the direction of the major axis. Theoretical ideas suggest two effects.

First, if an interplanetary field with an away polarity preferentially interconnects with northern dawn and southern dusk field lines, the field tension may twist the tail about the $X$ axis and thus rotate the cross tail current sheet. This is particularly likely if the normal component of the field across the magnetopause is the same at all azimuths around the tail cross section (COWLEY, 1981). Support for tail twisting was obtained by SIBECK et al. (1985, 1986b). The first paper investigated 12 hours of ISEE 3 data on January 15, 1983 when ISEE observed multiple crossings between the magnetosheath and the north lobe when the spacecraft was located below the solar ecliptic equatorial plane and the solar wind was flowing from the south. The second paper provided confirming evidence for twisting by noting that the detection of lobes with unexpected polarity for a given hemisphere was controlled by the IMF sector of appropriate polarity to support twisting. Also tail boundary crossings were associated with appropriate 
normal directions to support their case.

A second theoretical idea (MICHEL and DESSLER, 1970; SIBECK et al., 1986a; GRZEDZIELSKI and MACEK, 1988) is based on the proposition that interplanetary field lines will slip around the tail and create an anisotropic field pressure on the tail boundary. This effect involves inclusion of a $B^{2} / 8 \pi$ term on the left-hand side of Eq. (1) which is normally neglected in the subsolar region but which becomes important in the downstream region as the $\cos \theta$ term becomes small (e.g. MACEK, 1988). In the plane of the exterior field line there will be a point dividing field lines that slip over the tail from those that slip under the tail. At this point the field pressure on the tail will be zero. Where the IMF field direction is tangent to the tail the pressure will be maximum. SIBECK et al. (1986a) argue that the tail will tend to expand more in the direction parallel to the IMF where the exterior field pressure is small whereas it will be compressed more where the pressure is high, thus leading to an elliptical tail. Although this effect is not important near the earth where solar wind pressure effects dominate the pressure balance, further down the tail where the flaring angle approaches zero and the kinetic pressure becomes negligible relative to thermal and magnetic field pressure, this magnetosheath pressure effect may achieve increasing significance. SIBECK et al. (1986a) analyze ISEE 3 data and find support for this idea (1) in the relative frequency of time spent in the tail at different positions and (2) in the distribution of tail magnetopause normals. GRZEDZIELSKI and MACEK (1988) also find support for a flattened tail.

The diameter of the distant tail has also been a topic of considerable interest. On average, flaring of the distant magnetotail seems to terminate near $120 R_{E}$ with a "terminal diameter" of $30 \mathrm{R}_{\mathrm{E}}$ determined from ISEE 3 measurements (SLAviN et al., 1985). It should be noted however that these measurements are biased toward the equatorial plane and FAIRFIELD (1986) has argued that the average diameter may be nearer $25 R_{\mathrm{E}}$. The data of Sibeck et al. in Fig. 1 indicated an average diameter of about 25 $R_{E}$ near $40 R_{E}$ where the tail is still flaring, which raises the possibility that the distant tail might even be decreasing in diameter due to flux loss through the current sheet or magnetopause.

\section{Upstream Foreshock Effects on the Magnetopause}

Although simple theory would indicate that a solar wind of uniform density and velocity would exert a constant pressure on the magnetopause, recent observations have suggested that effects in the upstream foreshock region controlled by the IMF may complicate this picture (FAIRFIELD et al., 1990). Figure 7 shows three hours of data of September 12, 1984 when three spacecraft are measuring the magnetic field in the subsolar magnetosphere and AMPTE/IRM is monitoring the solar wind pressure just upstream from the bow shock. Magnetosphere field compressions appear to be related to solar wind pressure pulses of a few minutes duration on a one-to-one basis. Although this is not surprising, the authors go on to show that the solar wind pressure pulses are not inherent in the solar wind, but are associated with the foreshock. Apparently the density enhancements are generated in the foreshock and convected through the shock where they impinge on the magnetosphere. These processes seem to be related to readjustments in the foreshock associated with a changing IMF direction and represent a previouslyunappreciated mode of interaction between the solar wind and the magnetosphere.

In Fig. 7 the solar wind velocity also can be seen to be depressed by about 100 


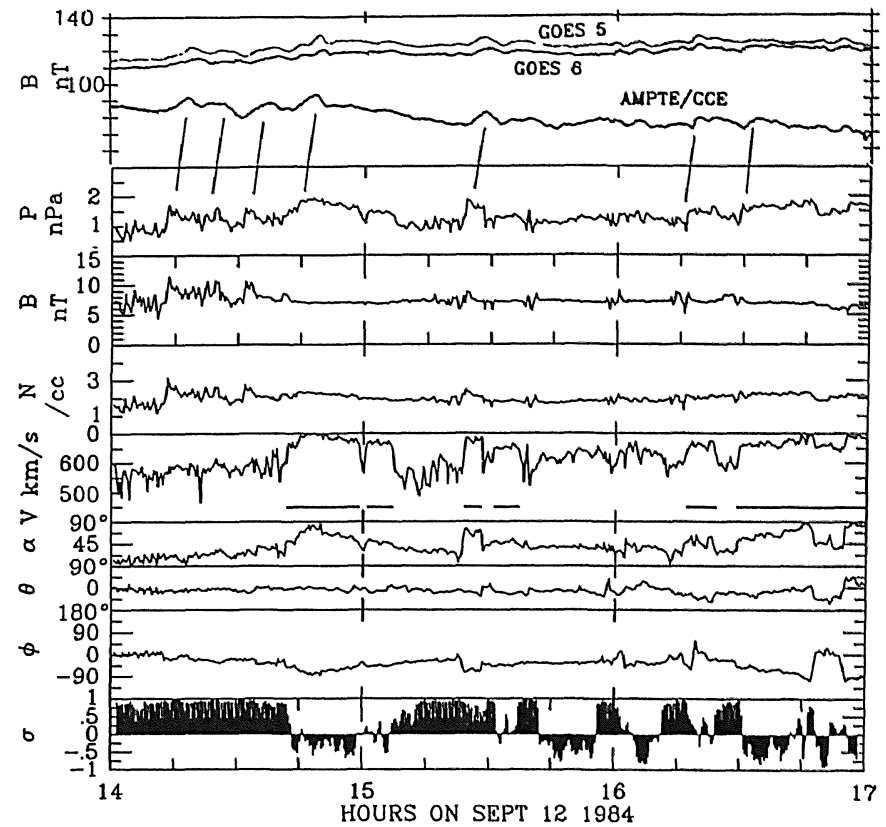

Fig. 7. Three hours of data when the GOES 5, GOES 6 and AMPTE/CCE spacecraft were measuring the magnetic field strength in the outer magnetosphere near the subsolar point (top panel) and AMPTE/IRM was measuring the solar wind pressure immediately upstream of the bow shock. Lower pancls show the solar wind kinetic pressure $P$ computed from the density $N$ and velocity $V$ along with the field strength $B$ and latitudinal and longitudinal directional angle 0 and $\phi$, the angle of the field makes with the carth-sun line $\alpha$, and a measure of running 2.4-min $B-n$ correlation $\sigma$. The pressure changes associated with magnetosphere field compressions are not inherent in the solar wind (FAIRFIELD et al., 1990).

$\mathrm{km} / \mathrm{sec}$ in the foreshock. The foreshock can be identified by high values of the correlation coefficient $\sigma$ between field strength and density caused by upstream waves (bottom panel) or by the bars under the velocity which indicate a lack of upstreaming energetic ions. Smaller velocity depressions in the foreshock have been previously reported (see references in FAIRFIELD et al., 1990). These data suggest that the usual supposition about the upstream solar wind not knowing about the presence of the earth is not strictly true. The authors suggest that the pressure on the magnetosphere may be dependent on the IMF direction through its control of the foreshock. This idea could also be couched in terms of an IMF dependence of $K$ in Eq. (1).

\section{Summary and Conclusions}

The size of the magnetosphere is clearly controlled by the solar wind pressure with the shape of the magnetopause being preserved for various solar wind pressures. The pressure exhibits a solar cycle variation in addition to day to day variations. The shape of the magnetosphere is effected by the IMF in a variety of ways: (1) A southward field clearly leads to a more earthward subsolar distance and a wider tail which is consistent with flux transfer to the tail during southward IMF conditions. (2) The shape of the cross 
section of the distant tail may be controlled by the IMF with the major axis of an elliptical tail aligned with the IMF field component in the $Y Z$ plane due to a weaker magnetosheath-magnetic field pressures in this direction. (3) The tail seems to be twisted by the torque associated with reconnected field lines. Suggestions that changes in the upstream foreshock may lead to pressure changes at the magnetopause should be pursued further.

The author thanks J. W. King for supplying the annual average solar wind parameters used in Fig. 3 and D. G. Sibeck for supplying a copy of his paper before publication. The comments of Sibeck and J. A. Slavin are also greatly appreciated.

\section{REFERENCES}

Aubry, M. P., C. T. RuSSELL, and M. G. KIVELSON, On inward motion of the magnetopause before a substorm J. Geophys. Res., 75, 7018-7031, 1970.

BURCH, J. L., Precipitation of low-energy electrons at high latitudes: Effects of interplanetary magnetic field and dipole tilt angle, J. Geophys. Res., 77, 6696-6707, 1972.

Cowl.EY, S. W. H., Magnetospheric asymmetries associated with the y-component of the IMF, Planet. Space Sci., 29, 79-96, 1981.

FAirfiEld, D. H., Average and unusual locations of the earth's magnetopause and bow shock, J. Geophys. Res., 76, 6700-6716, 1971.

FAIRFIELD, D. H., Global aspects of the earth's magnetopause, in Magnetospheric Boundary Layers, European Space Agency Scientific and Technical Publications Branch ESA SP-148, pp. 5-13, Noordwijk, The Netherlands, 1979.

FAIRFIELD, D. H., Solar wind control of magnetospheric pressure (CDAW 6), J. Geophys. Res., 90, 1201-1204, 1985.

FAirfield, D. H., Time variations of the distant magnetotail, Geophys. Res. Lett., 13, 80-83, 1986.

Fairfiel.d, D. H., D. N. BAker, J. D. Craven, R. C. Elphic, J. F. Fennell, L. A. Frank, l. G. Richardson, H. J. Singer, J. A. Slavin, B. T. Tsurutani, and R. D. Zwickl, Substorms, plasmoids, flux ropes, and magnetotail flux loss on March 25 1983: CDAW 8, J. Geophys. Res., 94, 15135-15152, 1989.

Fairfield, D. H., W. Baumjohann, G. Paschmann, H. Luehr, and D. G. Sibeck, Upstream pressure variations associated with the bow shock and their effects on the magnetosphere, J. Geophys. Res., 95, 3773-3786, 1990.

Formisano, V., V. Domingo, and K.-P. Wenzel, The three-dimensional shape of the magnetopause, Planet. Space Sci., 27, 1137-1149, 1979.

FrAnk, L. A. and J. D. CrAven, Imaging results from Dynamics Explorer I, Rev. Geophys., 26, 249-283, 1989.

GRZEDZILLSKI, S. and W. MACEK, An open magnetopause model of the Earth's distant tail based on ISEE 3 evidence, J. Geophys. Res., 93, 1795-1808, 1988.

Holizer, R. E. and J. A. St.Avin, Magnetic flux transfer associated with expansions and contractions of the dayside magnetosphere, J. Geophys. Res., 83, 3831-3839, 1978.

KING, J. H., Long-term solar wind variations and associated data sources, J. Geomag. Geoelectr., this supplement, 865-880, 1991.

MACEK, W., A study of long magnetic tails in the stellar wind plasma, Astron. Astrophys., 199, 329-339, 1988.

MAezıwı, K., Magnetotail boundary motion associated with geomagnetic substorms, J. Geophys. Res., 80, 3543-3548, 1975.

MAl.tsev, Yu. P. and W. B. LyAtsky, Field-aligned currents and erosion of the dayside magnetopause, Planet. Space Sci., 23, 1257-1260, 1975.

MiChel, F. C. and A. J. Dessler, Diffusive entry of solar flare particles into the geomagnetic tail, J. Geophys. Res., 75, 6061-6072, 1970.

Pizzo, V. J., The nature of the distant solar wind, Adv. Space Res., 6(1), 353-367, 1986.

RufenACH, C. L., R. F. MARTin, Jr., and H. H. SAuer, A study of geosynchronous magnetopause crossings, $J$. Geophys. Res., 94, 15125-15134, 1989.

RUSSEl.L, C. T., On the occurrence of magnetopause crossings at 6.6 $\mathrm{R}_{\mathrm{E}}$, Geophys. Res. Lett., 3, 593-595, 1976. 
Sibeck, D. G., G. L. Siscoe, J. A. Slavin, E. J. Smith, B. T. Tsurutani, and R. P. Lepping, The distant magnetotail's response to a strong interplanetary magnetic field $B_{y}$ : Twisting, flattening, and field line bending, J. Geophys. Res., 90, 4011-4019, 1985.

Sibeck, D. G., G. L. SisCoE, J. A. Slavin, and R. P. LepPiNG, Major flattening of the distant geomagnetic tail, J. Geophys. Res., 91, 4223-4237, 1986a.

Sibeck, D. G., J. A. Slavin, E. J. Smith, and B. T. Tsurutani, Twisting of the geomagnetic tail, in Solar Wind-Magnetosphere Coupling, edited by Y. Kamide and J. A. Slavin, pp. 731-738, Terra Sci.Publ. Co., Tokyo, 1986b.

Sibeck, D. G., R. E. Lopez, and E. C. Roelof, Solar wind control of the magnetopause shape, location and motion, J. Geophys. Res., 96, 5489-5495, 1991. 